\title{
Parameters of cosmological models and recent astronomical observations
}

\author{
G. S. Sharov ${ }^{1}$ and E. G. Vorontsova ${ }^{1}$ \\ ${ }^{1}$ Tver state university \\ 170002, Sadovyj per. 35, Tver, Russid*
}

\begin{abstract}
For different gravitational models we consider limitations on their parameters coming from recent observational data for type Ia supernovae, baryon acoustic oscillations, and from 34 data points for the Hubble parameter $H(z)$ depending on redshift. We calculate parameters of 3 models describing accelerated expansion of the universe: the $\Lambda$ CDM model, the model with generalized Chaplygin gas (GCG) and the multidimensional model of I. Pahwa, D. Choudhury and T.R. Seshadri. In particular, for the $\Lambda$ CDM model $1 \sigma$ estimates of parameters are: $H_{0}=70.262 \pm 0.319 \mathrm{~km} \mathrm{c}^{-1} \mathrm{Mpc}^{-1}, \Omega_{m}=0.276_{-0.008}^{+0.009}, \Omega_{\Lambda}=0.769 \pm 0.029$, $\Omega_{k}=-0.045 \pm 0.032$. The GCG model under restriction $\alpha \geq 0$ is reduced to the $\Lambda$ CDM model. Predictions of the multidimensional model essentially depend on 3 data points for $H(z)$ with $z \geq 2.3$.
\end{abstract}

\section{INTRODUCTION}

The most important challenge for cosmologists is to explain the accelerated expansion of our universe that was directly measured for the first time from Type Ia supernovae observations [1, 2]. These supernovae were used as standard candles, because one can measure their redshifts $z$ and luminosity distances $D_{L}$. The observed dependence $D_{L}(z)$ based on further measurements [3, 4] argues for the accelerated growth of the cosmological scale factor $a(t)$ at late stage of its evolution.

This result was confirmed via observations of cosmic microwave background anisotropy [5], baryon acoustic oscillations (BAO) or large-scale galaxy clustering [4, 6, 7] and other observations [4, 5, 8]. In particular, our attention should be paid to measurements of the Hubble parameter $H(z)$ for different redshifts $z$ [ 9 [20]. The results of these measurements and estimations are represented below in Table VI of Appendix.

The values $H(z)$ were calculated with two methods: evaluation of the age difference for galaxies with close redshifts in Refs. [9 15] and the method with BAO analysis [16 20].

In the first method the equality

$$
a(t)=a_{0} /(1+z)
$$

and its consequence

$$
H(z)=\frac{1}{a(t)} \frac{d a}{d t}=-\frac{1}{1+z} \frac{d z}{d t}
$$

are used. Here $a_{0} \equiv a\left(t_{0}\right)$ is the current value of the scale factor $a$.

Baryon acoustic oscillations (BAO) are disturbances in the cosmic microwave angular power spectrum and in the correlation function of the galaxy distribution, connected with acoustic waves propagation before the recombination epoch [4, 6]. These waves involved baryons coupled with photons up to the end of the drag era corresponding to $z_{d} \simeq 1059.3$ [8], when baryons became decoupled and resulted in a peak in the galaxy-galaxy correlation function at the comoving sound horizon scale $r_{s}\left(z_{d}\right)[6,8]$.

*Electronic address: german.sharov@mail.ru 
In Table $\nabla$ of Appendix we represent estimations of two observational manifestations of the BAO effect. These values are taken from Refs. [5, 21, 22], they confirm the conclusion about accelerated expansion of the universe. In addition, this data with observations of Type Ia supernovae and the Hubble parameter $H(z)$ are stringent restrictions on possible cosmological theories and models.

To explain accelerated expansion of the universe various cosmological models have been suggested, they include different forms of dark matter and dark energy in equations of state and various modifications of Einstein gravity [23 25]. The most popular among cosmological models is the $\Lambda$ CDM model with a $\Lambda$ term (dark energy) and cold dark matter (see reviews [23, 25]). This model with $5 \%$ fraction of visible baryonic matter nowadays $\left(\Omega_{b}=0.05\right), 24 \%$ fraction of dark matter $\left(\Omega_{c}=0.24\right)$ and $71 \%$ fraction of dark energy $\left(\Omega_{\Lambda}=0.71\right)[5]$ successfully describes observational data for Type Ia supernovae, anisotropy of cosmic microwave background, BAO effects and $H(z)$ estimates [4, 5, 8].

However, there are some problems in the $\Lambda \mathrm{CDM}$ model connected with vague nature of dark matter and dark energy, with fine tuning of the observed value of $\Lambda$, which is many orders of magnitude smaller than expected vacuum energy density, and with surprising proximity $\Omega_{\Lambda}$ and $\Omega_{m}=\Omega_{b}+\Omega_{c}$ nowadays, though these parameters depend on time in different ways (the coincidence problem) $[23$ 26].

Therefore a large number of alternative cosmological models have been proposed. They include modified gravity with $f(R)$ Lagrangian [27, 28], theories with scalar fields [29, 30], models with nontrivial equations of state [31 39], with extra dimensions [40 47] and many others [23 26].

Among these gravitational models we concentrate here on the model with generalized Chaplygin gas (GCG) 31 37]. The equation of state in this model

$$
p=-B_{0} / \rho^{\alpha}
$$

generalizes the corresponding equation $p=-B / \rho$ for the original Chaplygin gas model [31]. Generalized Chaplygin gas with EoS (2) plays the roles of both dark matter and dark energy, it is applied to describing observations of type Ia supernovae, BAO effects, the Hubble parameter $H(z)$ and other observational data in various combinations [33 37].

The equation of state similar to Eq. (2) is used in the multidimensional gravitational model of I. Pahwa, D. Choudhury and T.R. Seshadri [46] (the PCS model in references below). In this model the $1+3+d$ dimensional spacetime is symmetric and isotropic in two subspaces: in 3 usual spatial dimensions and in $d$ additional dimensions. Matter has zero (dust-like) pressure in usual dimensions and negative pressure $p_{e}$ in the form (2) in extra dimensions:

$$
T_{\nu}^{\mu}=\operatorname{diag}\left(-\rho, 0,0,0, p_{e}, \ldots, p_{e}\right), \quad p_{e}=-B_{0} \rho^{-\alpha}
$$

(in Sects. I. II] we use units with $c=1$ ).

In Ref. [46] the important case $d=1$ was omitted. This case was considered in Ref. [47], where we analyzed singularities of cosmological solutions in the PCS model [46] and suggested how to modify the equation of state (3) for the sake of avoiding the finite-time future singularity ("the end of the world") which is inevitable in the PCS model. Main advantages of the multidimensional models [46] and [47] are: naturally arising dynamical compactification and successful description of the Type Ia supernovae observations.

In this paper we compare the $\Lambda$ CDM model, the model with generalized Chaplygin gas (GCG) [31, 32], and also the models PCS [46] and [47] with $d$ extra dimensions from the point of view of their capacity to describe recent observational data for type Ia supernovae, BAO and $H(z)$. In the next section we briefly summarize the dynamics of the mentioned models, in Sect. III we analyze parameters of the mentioned models resulting in the best description of the observational data from Ref. [3] and Appendix. 


\section{MODELS}

For all cosmological models in this paper the Einstein equations

$$
G_{\nu}^{\mu}=8 \pi G T_{\nu}^{\mu}+\Lambda \delta_{\nu}^{\mu}
$$

determine dynamics of the universe. Here $T_{\nu}^{\mu}$ and $G_{\nu}^{\mu}=R_{\nu}^{\mu}-\frac{1}{2} R \delta_{\nu}^{\mu}$ are the energy momentum tensor and the Einstein tensor, $\Lambda$ is nonzero only in the $\Lambda$ CDM model. The energy momentum tensor has the form (3) in the multidimensional models [46, 47] and the standard form

$$
T_{\nu}^{\mu}=\operatorname{diag}(-\rho, p, p, p)
$$

in models with $3+1$ dimensions. In the $\Lambda$ CDM model baryonic and dark matter may be considered as one component of dust-like matter with density $\rho=\rho_{b}+\rho_{d m}$, so we suppose $p=0$ in Eq. (5). The fraction of relativistic matter (radiation and neutrinos) is close to zero for observable values $z \leq 2.3$. In the GCG model [31 37] pressure $p$ in the form (2) plays the role of dark energy, corresponding to the $\Lambda$ term in the $\Lambda$ CDM model.

For the Robertson-Walker metric with the curvature sign $k$

$$
d s^{2}=-d t^{2}+a^{2}(t)\left[\left(1-k r^{2}\right)^{-1} d r^{2}+r^{2} d \Omega\right]
$$

the Einstein equations (4) are reduced to the system

$$
\begin{aligned}
3 \frac{\dot{a}^{2}+k}{a^{2}} & =8 \pi G \rho+\Lambda, \\
\dot{\rho} & =-3 \frac{\dot{a}}{a}(\rho+p) .
\end{aligned}
$$

Eq. (8) results from the continuity condition $T_{\nu ; \mu}^{\mu}=0$, the dot denotes the time derivative.

Using the present time values of the Hubble constant and the critical density

$$
H_{0}=\left.\frac{\dot{a}}{a}\right|_{t=t_{0}}=\left.H\right|_{z=0}, \quad \rho_{c r}=\frac{3 H_{0}^{2}}{8 \pi G},
$$

we introduce dimensionless time $\tau$, densities $\bar{\rho}_{i}$, pressure $\bar{p}$ and logarithm of the scale factor [46, 47]:

$$
\tau=H_{0} t, \quad \bar{\rho}=\frac{\rho}{\rho_{c r}}, \quad \bar{\rho}_{b}=\frac{\rho_{b}}{\rho_{c r}}, \quad \bar{p}=\frac{p}{\rho_{c r}}, \quad \mathcal{A}=\log \frac{a}{a_{0}} .
$$

We denote derivatives with respect to $\tau$ as primes and rewrite the system (77), (8)

$$
\begin{aligned}
\mathcal{A}^{\prime}(\tau) & =\sqrt{\bar{\rho}+\Omega_{\Lambda}+\Omega_{k} e^{-2 \mathcal{A}}}, \\
\bar{\rho}^{\prime}(\tau) & =-3 \mathcal{A}^{\prime}(\bar{\rho}+\bar{p}) .
\end{aligned}
$$

Here

$$
\Omega_{m}=\frac{\rho\left(t_{0}\right)}{\rho_{c r}}, \quad \Omega_{\Lambda}=\frac{\Lambda}{3 H_{0}^{2}}, \quad \Omega_{k}=-\frac{k}{a_{0}^{2} H_{0}^{2}}
$$

are present time fractions of matter $\left(\Omega_{m}=\Omega_{b}+\Omega_{c}\right)$, dark energy and curvature in the equality

$$
\Omega_{m}+\Omega_{\Lambda}+\Omega_{k}=1
$$

resulting from Eq. (17) if we fix $t=t_{0}$. 
If we know an equation of state $\bar{p}=\bar{p}(\bar{\rho})$ for any model, we can solve the Cauchy problem for the system (11), (12) including initial conditions for variables (10) at the present epoch $t=t_{0}$ (here and below $t=t_{0}$ corresponds to $\tau=1$ )

$$
\left.\mathcal{A}\right|_{\tau=1}=0,\left.\quad \bar{\rho}\right|_{\tau=1}=\Omega_{m}
$$

In the $\Lambda$ CDM model Eq. (12) yields $\bar{\rho}=\Omega_{m} e^{-3 \mathcal{A}}=\Omega_{m}(1+z)^{3}$, so we solve only equation (11)

$$
\mathcal{A}^{\prime 2}=\frac{H^{2}}{H_{0}^{2}}=\Omega_{m} e^{-3 \mathcal{A}}+\Omega_{\Lambda}+\Omega_{k} e^{-2 \mathcal{A}} .
$$

with the first initial condition (15).

Equation (12) may be solved also and in the GCG model, but in this case we are to decompose all matter into two components [34 38]. One of these components is usual dust-like matter including baryonic matter; the other component is generalized Chaplygin gas with density $\rho_{g} \equiv \rho_{G C G}$ (and corresponding $\bar{\rho}_{g}=\rho_{g} / \rho_{c r}$ ). If the first component is pure baryonic and the latter describes both dark matter and dark energy, equations of state are:

$$
\bar{\rho}=\bar{\rho}_{b}+\bar{\rho}_{g}, \quad \bar{p}_{b}=0, \quad \bar{p}=\bar{p}_{g}=-B\left(\bar{\rho}_{g}\right)^{-\alpha}
$$

If we use the integrals $\bar{\rho}_{b}=\Omega_{b} e^{-3 \mathcal{A}}$ and $\bar{\rho}_{g}=\left[B+C e^{-3 \mathcal{A}(1+\alpha)}\right]^{1 /(1+\alpha)}$ of Eq. (12) for these components, equation (11) takes the form [33 38$]$

$$
\mathcal{A}^{\prime 2}=\frac{H^{2}}{H_{0}^{2}}=\Omega_{b} e^{-3 \mathcal{A}}+\left(1-\Omega_{b}-\Omega_{k}\right)\left[B_{s}+\left(1-B_{s}\right) e^{-3 \mathcal{A}(1+\alpha)}\right]^{1 /(1+\alpha)}+\Omega_{k} e^{-2 \mathcal{A}} .
$$

We solve this equation with the initial condition (15) $\left.\mathcal{A}\right|_{\tau=1}=0$. The dimensionless constant $B_{s}$ [37, 38] (it is denoted $A_{s}$ in Refs. [34, 35]) is expressed via $B$ or $B_{0}$ :

$$
B_{s}=B \cdot\left(1-\Omega_{b}-\Omega_{k}\right)^{-1-\alpha}, \quad B=B_{0} \rho_{c r}^{-1-\alpha} .
$$

For the multidimensional model PCS [46] and the model [47] in spacetime with $1+3+d$ dimensions the following metric is used [46]:

$$
d s^{2}=-d t^{2}+a^{2}(t)\left(\frac{d r^{2}}{1-k r^{2}}+r^{2} d \Omega\right)+b^{2}(t)\left(\frac{d R^{2}}{1-k_{2} R^{2}}+R^{2} d \Omega_{d-1}\right) .
$$

Here $b(t)$ and $k_{2}$ are the scale factor and curvature sign in extra dimensions (along with $a$ and $k$ for usual dimensions). For cosmological solutions in Refs. [46, 47] the scale factor $a(t)$ grows while $b(t)$ diminishes, in other words, some form of dynamical compactification [40 46] takes place, a size of compactified $b$ is small enough to play no essential role at the TeV scale.

In Refs. [46, 47] the authors considered only one component of their matter. Here we generalize these models and introduce the "usual" component with density $\bar{\rho}_{b}$ and the "exotic" component with $\bar{\rho}_{e}=\rho_{e} / \rho_{c r}$ and pressure $\bar{p}_{e}=p_{e} / \rho_{c r}$ in extra dimensions similarly to Eq. (17):

$$
\bar{\rho}=\bar{\rho}_{b}+\bar{\rho}_{e}, \quad \bar{p}_{e}=-B\left(\bar{\rho}_{e}\right)^{-\alpha}
$$

Dynamical equations for the models [46, 47] result from the Einstein equations (5) with $\Lambda=0$ and the energy momentum tensor (3), (21). In our notation (10) with $\mathcal{B}=\log \left(b / b_{0}\right)$ (where $\left.b_{0}=b\left(t_{0}\right)\right)$ these equations for $k_{2}=0$ and $d>1$ are [46, 47]

$$
\begin{aligned}
\mathcal{A}^{\prime \prime} & =\frac{1}{d+2}\left[d(d-1) \mathcal{B}^{\prime}\left(\frac{1}{2} \mathcal{B}^{\prime}-\mathcal{A}^{\prime}\right)-3(d+1) \mathcal{A}^{\prime 2}-3 d \bar{p}_{e}+(2 d+1) \Omega_{k} e^{-2 \mathcal{A}}\right], \\
\bar{\rho}_{b}^{\prime} & =-\bar{\rho}_{b}\left(3 \mathcal{A}^{\prime}+d \mathcal{B}^{\prime}\right), \quad \bar{\rho}_{e}^{\prime}=-3 \bar{\rho}_{e} \mathcal{A}^{\prime}-d\left(\bar{\rho}_{e}+\bar{p}_{e}\right) \mathcal{B}^{\prime}, \\
\mathcal{B}^{\prime} & =(d-1)^{-1}\left[-3 \mathcal{A}^{\prime}+\sqrt{3\left[(d+2) \mathcal{A}^{\prime 2}+2(d-1)\left(\bar{\rho}+\Omega_{k} e^{-2 \mathcal{A}}\right)\right] / d}\right] .
\end{aligned}
$$


If $d=1$ one should use [47]

$$
\mathcal{B}^{\prime}=\left(\bar{\rho}+\Omega_{k} e^{-2 \mathcal{A}}\right) / \mathcal{A}^{\prime}-\mathcal{A}^{\prime}
$$

instead of Eq. (24).

For the system (22) - (23) the initial conditions include Eqs. (15) and the additional condition

$$
\left.\mathcal{A}^{\prime}\right|_{\tau=1}=1
$$

resulting from definitions of $\mathcal{A}(10)$ and $H_{0}(9)$ :

$$
\mathcal{A}^{\prime}(\tau)=\frac{d}{d \tau} \log \frac{a}{a_{0}}=\frac{1}{H_{0}} \frac{\dot{a}}{a} .
$$

For the model PCS [46, 47] we have the analog of Eq. (14)

$$
\Omega_{m}+\Omega_{B}+\Omega_{k}=1,
$$

resulting from Eqs. (24) or (25) at $\tau=1$. Here $\Omega_{B}=-\left.d\left(B^{\prime}+\frac{d-1}{6} B^{\prime 2}\right)\right|_{\tau=1}$ is the contribution from $d$ extra dimensions.

The models $\Lambda$ CDM, GCG, PCS with suitable values of model parameters have cosmological solutions describing accelerated expansion of the universe [5, 8 , 33 37, 46, 47]. We consider restrictions on these parameters coming from recent observational data for type Ia supernovae [3], BAO [5, 21, 22] and from measuring the Hubble parameter $H(z)[9$ 20], (Tables V] VI).

\section{OBSERVATIONAL DATA AND MODEL PARAMETERS}

Recent observational data on Type Ia supernovae in the Union2.1 compilation [3] include redshifts $z=z_{i}$ and distance moduli $\mu_{i}$ with errors $\sigma_{i}$ for $N_{S}=580$ supernovae. The distance modulus $\mu_{i}=\mu\left(D_{L}\right)=5 \log \left(D_{L} / 10 \mathrm{pc}\right)$ is logarithm of the luminosity distance [8, 23]:

$$
D_{L}(z)=\frac{c(1+z)}{H_{0} \sqrt{\left|\Omega_{k}\right|}} \operatorname{Sin}_{k}\left(H_{0} \sqrt{\left|\Omega_{k}\right|} \int_{0}^{z} \frac{d \tilde{z}}{H(\tilde{z})}\right), \quad \operatorname{Sin}_{k}(x)= \begin{cases}\sinh x, & \Omega_{k}>0 \\ x, & \Omega_{k}=0 \\ \sin x, & \Omega_{k}<0\end{cases}
$$

In particular, for the flat universe $\left(k=\Omega_{k}=0\right)$ the expression (28) is

$$
D_{L}=c(1+z) \int_{0}^{z} \frac{d \tilde{z}}{H(\tilde{z})}=\frac{c a_{0}^{2}}{H_{0} a(\tau)} \int_{\tau}^{1} \frac{d \tilde{\tau}}{a(\tilde{\tau})}
$$

To describe the Type Ia supernovae data [3] we fix values of model parameters $p_{1}, p_{2}, \ldots$ for the chosen model $\Lambda \mathrm{CDM}, \mathrm{GCG}$ or PCS and calculate dependence of the scale factor $a(\tau)$ on dimensionless time $\tau$. Further, we calculate numerically the integral expression (28) and the distance modulus $\mu(\tau)$. For each value of redshift $z_{i}$ in the table [3] we find the corresponding $\tau=\tau_{i}$ with using linear approximation in Eq. (11) and the theoretical value $\mu_{t h}=\mu\left(\tau_{i}, p_{1}, p_{2}, \ldots\right)$ from the dependence $\mu(\tau)$ (28).

We search a good fit between theoretical predictions $\mu_{t h}$ and the observed data $\mu_{i}$ as the minimum of

$$
\chi_{S}^{2}\left(p_{1}, p_{2}, \ldots\right)=\sum_{i=1}^{N_{S}} \frac{\left[\mu_{i}-\mu_{t h}\left(z_{i}, p_{1}, p_{2}, \ldots\right)\right]^{2}}{\sigma_{i}^{2}}
$$


or the maximum of the corresponding likelihood function $\mathcal{L}_{S}\left(p_{1}, p_{2}, \ldots\right)=\exp \left(-\chi_{S}^{2} / 2\right)$ in the space of model parameters $p_{1}, p_{2}, \ldots$

The Type Ia supernovae data [3] and the best fits for the mentioned models $\Lambda$ CDM, GCG and PCS are shown in Fig. 1 $\mathrm{b}$ in $z, D_{L}$ plane. Details of the optimization procedure are described below.

Model predictions for the Hubble parameter $H(z)=\dot{a} / a=H_{0} \mathcal{A}^{\prime}(\tau)$ we compare with observational data [9 20], from Table VI (Fig. 15) and use the $\chi^{2}$ function similar to (29):

$$
\chi_{H}^{2}\left(p_{1}, p_{2}, \ldots\right)=\sum_{i=1}^{N_{H}} \frac{\left[H_{i}-H_{t h}\left(z_{i}, p_{1}, p_{2}, \ldots\right)\right]^{2}}{\sigma_{H, i}^{2}} .
$$

Here $N_{H}=34$, theoretical values $H_{t h}\left(z_{i}, \ldots\right)=H_{0} \mathcal{A}^{\prime}\left(\tau\left(z_{i}\right)\right)$ are obtained from the calculated dependence $\mathcal{A}(\tau)$ and the equality (11) $z=e^{-\mathcal{A}}-1$.

The observational data for BAO [5, 21, 22] (Table V) includes two measured values [6]

$$
d_{z}(z)=\frac{r_{s}\left(z_{d}\right)}{D_{V}(z)}
$$

and

$$
A(z)=\frac{H_{0} \sqrt{\Omega_{m}}}{c z} D_{V}(z) .
$$

They are connected with the distance [5, 6, [8]

$$
D_{V}(z)=\left[\frac{c z D_{L}^{2}(z)}{(1+z)^{2} H(z)}\right]^{1 / 3}
$$

expressed here via the luminosity distance (28).

The BAO observations [5, 21, 22] in Table $\mathrm{V}$ are not independent. So the $\chi^{2}$ function for the values (31) and (32)

$$
\chi_{B}^{2}\left(p_{1}, p_{2}, \ldots\right)=(\Delta d)^{T} C_{d}^{-1} \Delta d+(\Delta A)^{T} C_{A}^{-1} \Delta A .
$$

includes the columns $\Delta d=\left[d_{z, t h}\left(z_{i}, p_{1}, \ldots\right)-d_{z}\left(z_{i}\right)\right], \Delta A=\left[A_{t h}\left(z_{i}, p_{1}, p_{2}, \ldots\right)-A\left(z_{i}\right)\right], i=$ $1, \ldots, N_{B}$ and the covariance matrices $C_{d}^{-1}$ and $C_{A}^{-1}[5,21]$ described in Appendix.

The best fits to the observational data for Type Ia supernovae [3], $H(z)$ and BAO data from Tables V, VI are presented in Fig. 1 for the models $\Lambda$ CDM, GCG and PCS (with $d=1$ and $d=6$ ). The values of model parameters are tabulated below in Table II They are optimal from the standpoint of minimizing the sum of all $\chi^{2}$ (29), (30) and (34):

$$
\chi_{\Sigma}^{2}=\chi_{S}^{2}+\chi_{H}^{2}+\chi_{B}^{2} .
$$

Predictions of different models in Fig. 1 1 are rather close, in particular, the curves for the models $\Lambda \mathrm{CDM}$ and GCG practically coincide. The Hubble parameter $H(z)$ in Fig. 1c is measured in $\mathrm{km} \mathrm{c}^{-1} \mathrm{Mpc}^{-1}$, the distances $D_{L}(z)$ and $D_{V}(z)$ in Fig. 1 b, d are in Gpc.

The data points for $D_{V}(z)=r_{s}\left(z_{d}\right) / d_{z}(z)$ in Fig. 1 1 d are calculated from $d_{z}\left(z_{i}\right)$ in Table $\mathrm{V}$. Here the error boxes include the data spread between the recent estimations of the comoving sound horizon size:

$$
r_{s}\left(z_{d}\right)=147.49 \pm 0.59 \mathrm{Mpc} \text { [8], } \quad r_{s}\left(z_{d}\right)=153.3 \pm 2.0 \mathrm{Mpc} \text { [17, 21]. }
$$



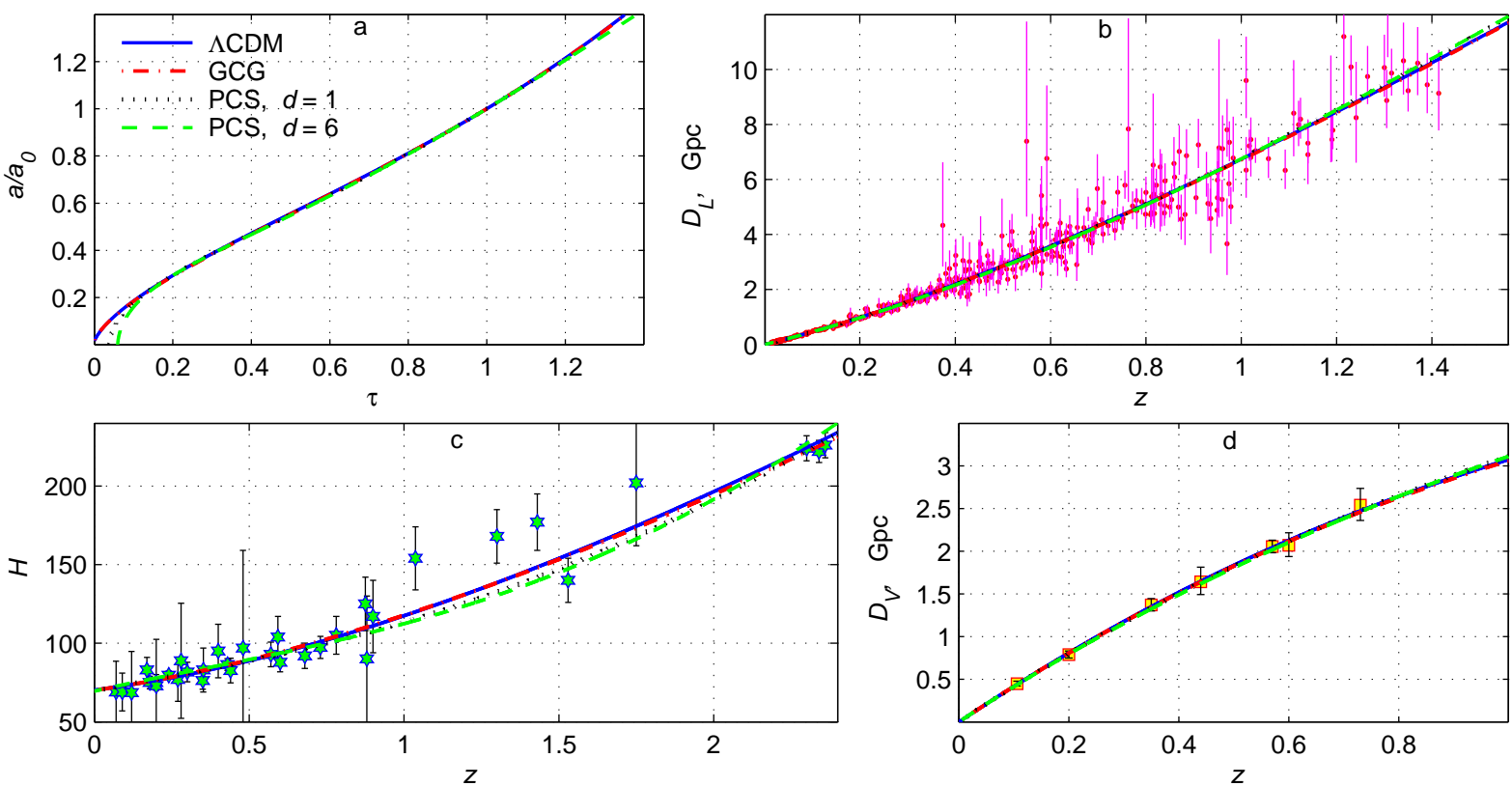

FIG. 1: For the models $\Lambda$ CDM, GCG, PCS $(d=1$ and $d=6)$ with the optimal values of model parameters from Table II we present (a) the scale factor $a(\tau)$; (b) the luminosity distance $D_{L}(z)$ and the Type Ia supernovae data [3]; (c) dependence $H(z)$ with the data points from Table VI and (d) the distance (33) $D_{V}(z)$ with the data points from Table $\mathrm{V}$

\section{A. $\Lambda$ CDM model}

In the $\Lambda \mathrm{CDM}$ model we use three free parameters $H_{0}, \Omega_{m}$ and $\Omega_{\Lambda}$ in Eq. (16) for describing the considered observational data at $z \leq 2.3$. For the Hubble constant $H_{0}$ different approaches result in different estimations. In particular, observations of Cepheid variables in the project Hubble Space Telescope (HST) give the recent estimate $H_{0}=73.8 \pm 2.4 \mathrm{~km} \mathrm{c}^{-1} \mathrm{Mpc}^{-1}$ [48]. On the other hand, the satellite projects Planck Collaboration (Planck) [8] and Wilkinson Microwave Anisotropy Probe (WMAP) [5] for observations of cosmic microwave background anisotropy result in the following values (in $\mathrm{km} \mathrm{c}^{-1} \mathrm{Mpc}^{-1}$ ):

$$
\begin{array}{ll}
H_{0}=67.3 \pm 1.2 & (\text { Planck [8]) }, \\
H_{0}=69.7 \pm 2.4 \quad(\text { WMAP [5]) } & \\
H_{0}=73.8 \pm 2.4 & (\text { HST [48] }) .
\end{array}
$$

The nine-year results from WMAP [5] include also the estimate $H_{0}=69.33 \pm 0.88 \mathrm{~km} \mathrm{c}^{-1} \mathrm{Mpc}^{-1}$ with added recent $\mathrm{BAO}$ and $H_{0}$ observations.

For the $\Lambda$ CDM model many authors $[5,8,49,55]$ calculated the best fits for parameters $H_{0}, \Omega_{m}$ and $\Omega_{\Lambda}$ for describing the Type Ia supernovae, $H(z)$ and BAO data in various combinations. In Refs. 52 55] some other cosmological models were compared with the $\Lambda$ CDM model. In particular, the authors [52] compared 8 models with two information criteria including minimal $\chi^{2}$ and the number of model parameters. Optimal values of these parameters were pointed out in Ref. 52] with the exception of $H_{0}$, though $H_{0}$ is the important parameter for all 8 models.

In Refs. [53 55] the $\Lambda$ CDM, XCDM and $\phi \mathrm{CDM}$ models were applied to describe the supernovae, $H(z)$ and BAO data. For all mentioned models the authors [53 55] fixed two values of the Hubble constant $H_{0}=68 \pm 2.8$ [56] and $H_{0}=73.8 \pm 2.4 \mathrm{~km} \mathrm{c}^{-1} \mathrm{Mpc}^{-1}$ [48] and searched optimal values of other model parameters. But they did not estimated the best choice of $H_{0}$ among these two values and in the segment between them. 
In this paper we pay the special attention to dependence of $\chi_{\Sigma}^{2}$ minima on $H_{0}$. This dependence is very important if we compare different cosmological models.

The results of calculations [5, 8, 51 [55], as usual, are presented as level lines for the functions $\chi^{2}\left(p_{1}, p_{2}\right)$ or $\mathcal{L}_{S}\left(p_{1}, p_{2}\right)=\exp \left(-\chi_{S}^{2} / 2\right)$ of two parameters at $1 \sigma(68.27 \%), 2 \sigma(95.45 \%)$ and $3 \sigma$ $(99.73 \%)$ confidence levels. In particular, if a value $H_{0}$ is fixed, these two parameters for the $\Lambda$ CDM model may be $\Omega_{m}$ and $\Omega_{\Lambda}$.

In Fig. 2 we use this scheme for 3 fixed values $H_{0}$ (37) indicated on the panels (including the optimal value $H_{0}=70.262 \mathrm{~km} \mathrm{c}^{-1} \mathrm{Mpc}^{-1}$ ) and draw level lines of the functions (29), (30), (34) and (35) $\chi^{2}\left(\Omega_{m}, \Omega_{\Lambda}\right)$ in the $\Omega_{m}, \Omega_{\Lambda}$ plane and for $\chi_{\Sigma}^{2}\left(\Omega_{m}, H_{0}\right)$ with fixed $\Omega_{\Lambda}=0.769$ in the bottomright panel. The points of minima are marked in Fig. 2 as hexagrams for $\chi_{S}^{2}$, pentagrams for $\chi_{H}^{2}$, diamonds for $\chi_{B}^{2}$ and circles for $\chi_{\Sigma}^{2}$. Minimal values of the functions $\chi^{2}$ (29), (30), (34) and (35) at these points are tabulated in Table \so we can compare efficiency of this description for different $H_{0}$. For the same purpose we point out the corresponding values $\chi^{2}$ for some level lines in Fig. 2 and present the dependence of minima $\min \chi_{\Sigma}^{2}$ on $H_{0}$ and on $\Omega_{m}$ in the left bottom panels of Fig. 2, Here we denote $\min \chi_{\Sigma}^{2}\left(H_{0}\right)=\min _{\Omega_{m}, \Omega_{\Lambda}} \chi_{\Sigma}^{2}, \min \chi_{\Sigma}^{2}\left(\Omega_{m}\right)=\min _{H_{0}, \Omega_{\Lambda}} \chi_{\Sigma}^{2}$ and graphs of the fractions $\chi_{S}^{2}$, $\chi_{H}^{2}, \chi_{B}^{2}$ in $\min \chi_{\Sigma}^{2}\left(H_{0}\right)$ are also shown.

In the bottom panels we present how parameters of a minimum point of $\chi_{\Sigma}^{2}$ depend on $H_{0}$ and on $\Omega_{m}$. In particular, for the dependence on $H_{0}$ the coordinates $\Omega_{m}\left(H_{0}\right)$ and $\Omega_{\Lambda}\left(H_{0}\right)$ of this point are calculated, the value $\Omega_{k}$ is determined from Eq. (14). For the dependence on $\Omega_{m}$ we also present the graph $h\left(\Omega_{m}\right)$, where $h=H_{0} / 100$.

We see in Fig. 2 and in Table $\prod$ that the dependence of $\min \chi_{\Sigma}^{2}\left(H_{0}\right)$ is appreciable and significant. This function has the distinct minimum and achieves its minimal value 585.35 at $H_{0} \simeq 70.26$. The optimal values of the $\Lambda \mathrm{CDM}$ model parameters $\Omega_{m} \simeq 0.276, \Omega_{\Lambda} \simeq 0.769$, corresponding to this minimum are presented in Table II, these values are taken for the $\Lambda$ CDM curves in Fig. 1.

The mentioned sharp dependence of $\min \chi_{\Sigma}^{2}$ on $H_{0}$ is connected with two factors: (1) the similar dependence of the main contribution $\chi_{S}^{2}\left(H_{0}\right)$ shown in the same panel; (2) the large shift of the minimum point for $\chi_{S}^{2}$ in the $\Omega_{m}, \Omega_{\Lambda}$ plane corresponding to $H_{0}$ growth. For $H_{0}=68$ and 73.8 $\mathrm{km} \mathrm{c}^{-1} \mathrm{Mpc}^{-1}$ this minimum point is far from the similar points of $\chi_{H}^{2}$ and $\chi_{B}^{2}$. Only for $H_{0}$ close to $70 \mathrm{~km} \mathrm{c}^{-1} \mathrm{Mpc}^{-1}$ all these three minimum points are near each other (the top-right panel in Fig. 2).

Only the value $H_{0}=69.7 \mathrm{~km} \mathrm{c}^{-1} \mathrm{Mpc}^{-1}$ in Table I is close to the optimal value in Table II We may conclude that the values of the Hubble constant $H_{0}=68$ and $73.8 \mathrm{~km} \mathrm{c}^{-1} \mathrm{Mpc}^{-1}$ taken in Refs. [53 55], unfortunately, lie to the left and to the right from the optimal value $H_{0} \simeq 70$ $\mathrm{km} \mathrm{c}^{-1} \mathrm{Mpc}^{-1}$. We see the significant difference between the large values $\min \chi_{\Sigma}^{2}=673.64$ or 707.84 for the too small and too large values of $H_{0}$ in Table $\llbracket$ and the optimal value $\min \chi_{\Sigma}^{2}=585.35$ for $H_{0}=70.262$ in Table I.

In the middle row panels of Fig. 2 with $\chi_{\Sigma}^{2}$ the flatness line $\Omega_{m}+\Omega_{\Lambda}=1$ (or $\Omega_{k}=0$ ) is shown as the black dashed straight line. This line shows that only for $H_{0}$ close to the optimal value from Table II the following recent observational limitations on the $\Lambda$ CDM model parameters (13) from surveys [5, 8]

$$
\begin{array}{llll} 
& \Omega_{m}=0.279 \pm 0.025, & & \Omega_{m}=0.314 \pm 0.02 \\
\text { WMAP [5]: } & \Omega_{\Lambda}=0.721 \pm 0.025, \\
& \Omega_{k}=-0.0027_{-0.0038}^{+0.0039} ; \quad \text { Planck [8]: } & \Omega_{\Lambda}=0.686 \pm 0.025 \\
& \Omega_{k}=-0.0005_{-0.0066}^{+0.0065}
\end{array}
$$

are satisfied on $1 \sigma$ or $2 \sigma$ level. For $H_{0}=67.3$ and $73.8 \mathrm{~km} \mathrm{c}^{-1} \mathrm{Mpc}^{-1}$ the optimal values of parameters $\Omega_{m}, \Omega_{\Lambda}, \Omega_{k}$ in Table \are far from restrictions (38) for $\Omega_{k}$ even on $3 \sigma$ level.

Graphs of the optimal values $\Omega_{m}, \Omega_{\Lambda}$ and $\Omega_{k}$ depending on $H_{0}$ are presented in the second bottom panel. We see that the value $\Omega_{m}$ weakly depends on $H_{0}$, but $\Omega_{\Lambda}$ and $\Omega_{k}$ satisfy conditions 

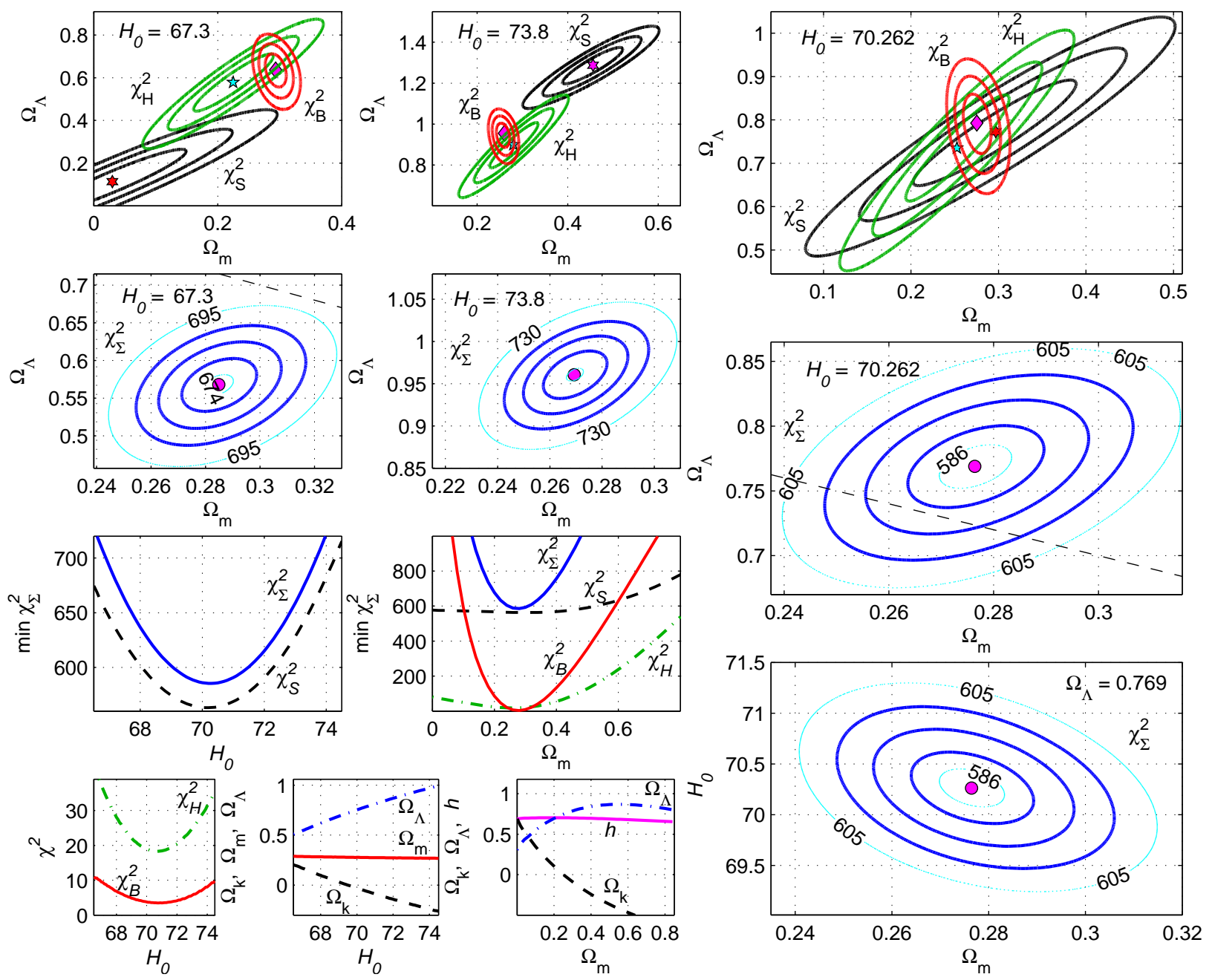

FIG. 2: The $\Lambda$ CDM model. For the values $H_{0}$ (37) and the optimal value $H_{0}=70.26 \mathrm{~km} \mathrm{c}^{-1} \mathrm{Mpc}^{-1}$ level lines are drawn at $1 \sigma, 2 \sigma$ and $3 \sigma$ (thick solid) for $\chi_{S}^{2}\left(\Omega_{m}, \Omega_{\Lambda}\right)$ (black), for $\chi_{H}^{2}\left(\Omega_{m}, \Omega_{\Lambda}\right)$ (green) and $\chi_{B}^{2}\left(\Omega_{m}, \Omega_{\Lambda}\right)$ (red in the top row), the sum (35) $\chi_{\Sigma}^{2}\left(\Omega_{m}, \Omega_{\Lambda}\right)$ (the middle row), $\chi_{\Sigma}^{2}\left(\Omega_{m}, H_{0}\right)$ for $\Omega_{\Lambda}=0.758$ (the bottom-right panel); dependence of $\min \chi_{\Sigma}^{2}$, its fractions $\chi^{2}$ and parameters of a minimum point on $H_{0}$ and on $\Omega_{m}$.

TABLE I: The $\Lambda$ CDM model. For given $H_{0}$ (37) the calculated minima of $\chi_{S}^{2}, \chi_{H}^{2}, \chi_{B}^{2}$ and $\chi_{\Sigma}^{2}$ with $\Omega_{m}$, $\Omega_{\Lambda}, \Omega_{k}$ correspondent to $\min \chi_{\Sigma}^{2}$.

\begin{tabular}{||c||c|c|c||c|c|c|c||}
\hline$H_{0}$ & $\min \chi_{S}^{2}$ & $\min \chi_{H}^{2}$ & $\min \chi_{B}^{2}$ & $\min \chi_{\Sigma}^{2}$ & $\Omega_{m}$ & $\Omega_{\Lambda}$ & $\Omega_{k}$ \\
\hline 67.3 & 599.37 & 18.492 & 5.548 & 673.64 & 0.285 & 0.568 & 0.147 \\
\hline 69.7 & 562.73 & 17.993 & 3.517 & 588.53 & 0.278 & 0.734 & -0.012 \\
\hline 73.8 & 639.90 & 19.466 & 5.322 & 707.84 & 0.269 & 0.961 & -0.230 \\
\hline
\end{tabular}

(38) only for $H_{0} \simeq 70 \mathrm{~km} \mathrm{c}^{-1} \mathrm{Mpc}^{-1}$.

The dependence of $\min _{H_{0}, \Omega_{\Lambda}} \chi_{\Sigma}^{2}$ on $\Omega_{m}$ is rather sharp because of the correspondent dependence of its fraction $\chi_{B}^{2}$. This fact for $\chi_{B}^{2}$ is connected with the contribution from the value $A(z)$ (32) measurements, because $A(z)$ is proportional to $\sqrt{\Omega_{m}}$ and $\chi_{B}^{2}$ is very sensitive to $\Omega_{m}$ values. Note that the fractions $\chi_{S}^{2}$ and $\chi_{H}^{2}$ (in min $\chi_{\Sigma}^{2}$ ) weakly depend on $\Omega_{m}$. 
Dependencies of $\min \chi_{\Sigma}^{2}$ on $H_{0}, \Omega_{m}$ and also $\Omega_{\Lambda}, \Omega_{k}$ let us calculate estimates of acceptable values for these model parameters. They are presented below in Table III.

Coordinates $h=H_{0} / 100$ and $\Omega_{\Lambda}$ of the minimum point for $\chi_{\Sigma}^{2}$ depend on $\Omega_{m}$ in a such manner that only for $\Omega_{m} \simeq 0.27$ values $\Omega_{\Lambda}$ and $\Omega_{k}$ satisfy conditions (38). Note that the optimal value of $h$ is close to 0.7 for all $\Omega_{m}$ in the limits $0<\Omega_{m}<1$.

\section{B. GCG model}

Let us apply the model with generalized Chaplygin gas (GCG) 31 37] to describing the same observational data for Type Ia supernovae, $H(z)$ and BAO. We use here Eq. (18) with the initial condition $\left.\mathcal{A}\right|_{\tau=1}=0$, so we have 5 independent free parameters in this model: $H_{0}, \Omega_{b}, \Omega_{k}, \alpha$ and $B_{s}$. However we really used only 4 free parameters, because the fraction $\Omega_{b}$ may include not only baryonic but also a part of cold dark matter. Our calculations yield that the minimum over remaining 4 parameters $\min _{H_{0}, \Omega_{k}, \alpha, B_{s}} \chi_{\Sigma}^{2}$ practically does not depend on $\Omega_{b}$ in the range $0 \leq \Omega_{b} \leq 0.25$ (see Fig. 3). So in our analysis presented in Fig. 3 (except for 3 bottom-right panels) we fixed the value

$$
\Omega_{b}=0.047,
$$

that is the simple average of the WMAP $\Omega_{b}=0.0464$ [5] and Planck $\Omega_{b}=0.0485$ [8] estimations.

In the GCG model $\Omega_{\Lambda}=0$ and $\Omega_{m}=1-\Omega_{k}$ in accordance with Eq. (14) and the formal definition (13). However we should use the effective value $\Omega_{m}^{e f f}$ in this model, in particular, in expression (32). In Refs. [34 38] the following effective value is used

$$
\Omega_{m}^{e f f}=\Omega_{b}+\left(1-\Omega_{b}-\Omega_{k}\right)\left(1-B_{s}\right)^{1 /(1+\alpha)} .
$$

This value results from correspondence between the models $\Lambda$ CDM with Eq. (16) and GCG with Eq. (18) in the early universe at $z \gg 1$.

But in our investigation the majority of observational data is connected with redshifts $0<z<1$, so in Eq. (32) we are to consider the present time limit of the value $\Omega_{m}^{e f f} \equiv \Omega_{0 m}^{e f f}=\lim _{z \rightarrow 0} \Omega_{m}^{e f f}$. If we compare limits of the right hand sides of Eqs. (16) and (18) at $z \rightarrow 0$ or $\mathcal{A} \rightarrow 0$, we obtain another effective value

$$
\Omega_{m}^{e f f}=\Omega_{b}+\left(1-\Omega_{b}-\Omega_{k}\right)\left(1-B_{s}\right)
$$

Values $\chi_{B}^{2}$ calculated with expressions (39) and (40) are different if $\alpha \neq 0$. This difference looks like rather small if we compare minima of the sum (35) $\min \chi_{\Sigma}^{2}=\min _{\Omega_{k}, \alpha, B_{s}} \chi_{\Sigma}^{2}$ depending on $H_{0}$. In Fig. 3 this dependence with Eq. (40) for $\Omega_{m}^{e f f}$ is the blue solid line and for the case with Eq. (39) it is the violet dash-and-dot line. We see that the lines closely converge in the vicinity of the minimum point $H_{0} \simeq 70 \mathrm{~km} \mathrm{c}^{-1} \mathrm{Mpc}^{-1}$. The dependence $\min \chi_{\Sigma}^{2}\left(H_{0}\right)$ in both cases (39) and (40) has the sharp minimum and resembles the case of the $\Lambda$ CDM model in Fig. 2, The value $\min \chi_{\Sigma}^{2} \simeq 584.54$ of this minimum, its parameters in Table II graph of the contribution $\chi_{S}^{2}$ and dependence on $H_{0}$ for parameters $\alpha, \Omega_{k}, B_{s}$ of the minimum point in the bottom-left panel in Fig. 3 are presented for the case with Eq. (40).

One should note that all mentioned dependencies are different for the case (39), in particular, the absolute minimum of $\chi_{\Sigma}^{2}$ is 584.31. This difference is illustrated in the central panels in Fig. 3 with level lines of $\chi_{\Sigma}^{2}\left(\alpha, B_{s}\right)$ for $H_{0}=73.8$ and $70.093 \mathrm{~km} \mathrm{c}^{-1} \mathrm{Mpc}^{-1}$ (with the specified values $\Omega_{k}$, optimal for these $H_{0}$ ). These level lines are blue for the expression (40) and they are thin violet 
for Eq. (39) . Positions of the optimal points are close only if $H_{0}$ is close to its optimal value in Table II.

We suppose that the estimation of $\chi_{B}^{2}$ with the expression (40) is more adequate to the considered values $z$. So in Table II and in other panels of Fig. 3 we use only Eq. (40). Notations in Fig. 3 correspond to Fig. 2 .
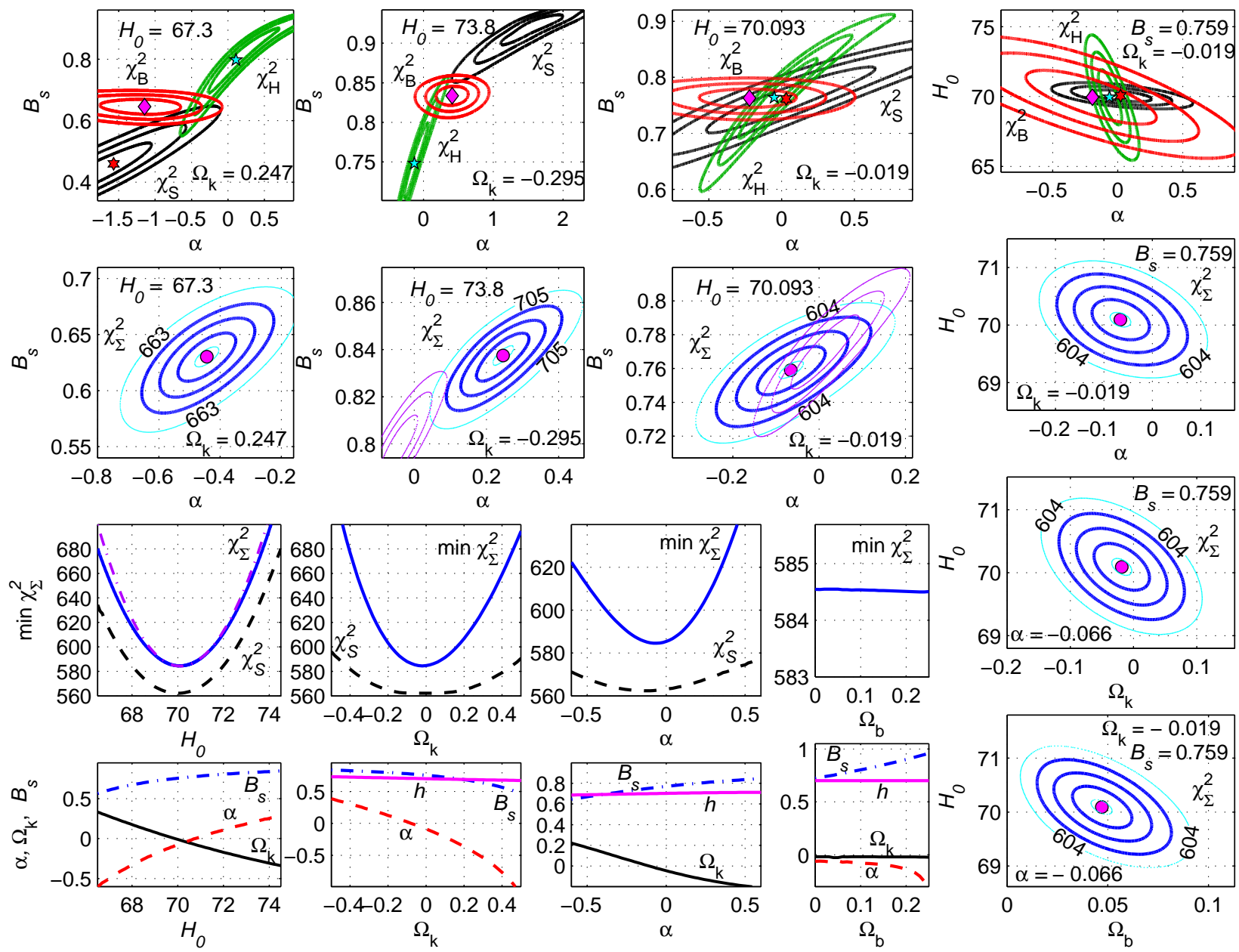

FIG. 3: The GCG model. For $H_{0}$ (37) and the optimal value $H_{0}=70.093 \mathrm{~km} \mathrm{c}^{-1} \mathrm{Mpc}^{-1}$ level lines of $\chi_{\Sigma}^{2}$ and other $\chi^{2}$ are presented in $\alpha, B_{s} ; \alpha, H_{0} ; \Omega_{k}, H_{0}$ and $\Omega_{b}, H_{0}$ planes in notations of Fig. 2. In the bottom-left panels we analyze dependence of $\min \chi_{\Sigma}^{2}$ and parameters of a minimum point on $H_{0}, \Omega_{k}, \alpha$ and $\Omega_{b}$.

The similar dependence of min $\chi_{\Sigma}^{2}$ on $H_{0}$ for the $\Lambda$ CDM and GCG models results in unsuccessful description of the data with $H_{0}=67.3$ and $73.8 \mathrm{~km} \mathrm{c}^{-1} \mathrm{Mpc}^{-1}$ with the corresponding optimal values $\Omega_{k}=0.247$ and -0.295 . Fig. 3 illustrates large distances between minimum points of $\chi_{S}^{2}$, $\chi_{H}^{2}$ and $\chi_{B}^{2}$ in these cases. The mentioned distances are small for the optimal values from Table II $H_{0}=70.093 \mathrm{~km} \mathrm{c}^{-1} \mathrm{Mpc}^{-1}$ and $\Omega_{k}=-0.19$. For these optimal values we present level lines of $\chi_{\Sigma}^{2}$ in $\alpha, B_{s} ; \alpha, H_{0} ; \Omega_{k}, H_{0}$ and $\Omega_{b}, H_{0}$ planes. In these panels other model parameters are fixed and specified.

When we test dependence of the minimum $\min \chi_{\Sigma}^{2}$ on $H_{0}, \Omega_{k}, \alpha$ and $\Omega_{b}$ in Fig. [3, we minimize this value over all other parameters (except for the above mentioned $\Omega_{b}$ ). In particular, $\min \chi_{\Sigma}^{2}\left(\Omega_{k}\right)=\min _{H_{0}, \alpha, B_{s}} \chi_{\Sigma}^{2}$, this function has the distinct minimum near $\Omega_{k} \simeq 0$ and resembles the 
dependence $\min \chi_{\Sigma}^{2}\left(H_{0}\right)$. The optimal value of $H_{0}$ or $h=H_{0} / 100$ is practically constant and close to $h \simeq 0.7$ if we vary $\Omega_{k}, \alpha$ or $\Omega_{b}$. As mentioned above the dependence of $\min \chi_{\Sigma}^{2}$ on $\Omega_{b}$ is very weak, so we fixed in our previous analysis $\Omega_{b}=0.047$.

For the graph $\min \chi_{\Sigma}^{2}(\alpha)=\min _{H_{0}, \Omega_{k}, B_{s}} \chi_{\Sigma}^{2}$ the correspondent minimum is achieved if $\alpha$ is negative: $\alpha=-0.066$ (see Table III). In the GCG model this parameter is connected with the square of adiabatic sound speed [33, 36, 37]

$$
c_{s}^{2}=\frac{\delta p}{\delta \rho}=-\alpha \frac{p}{\rho}
$$

If we accept the restriction $\alpha \geq 0$ (equivalent to $c_{s}^{2} \geq 0$ ) in our investigation with the mentioned observational data, we obtain the optimal value $\alpha=0$ and the GCG model will be reduced to the $\Lambda$ CDM model with $\Omega_{\Lambda}=B=B_{s}\left(1-\Omega_{b}-\Omega_{k}\right)$. The dependence of min $\chi_{\Sigma}^{2}$ and other parameters on $\alpha$ in Fig. 3 show that for $\alpha=0$ we have $\min \chi_{\Sigma}^{2} \simeq 585.35$ and the optimal values of $H_{0}, \Omega_{k}$, $\Omega_{\Lambda}=B$ corresponding to the $\Lambda$ CDM model in Table $\amalg$

TABLE II: Optimal values of model parameters $\left(\Omega_{b}=0.047\right.$, for the GCG model $\Omega_{m}=\Omega_{m}^{e f f}$ (40)).

\begin{tabular}{||l||c||c|c|ccc||}
\hline Model & $\min \chi_{\Sigma}^{2}$ & $H_{0}$ & $\Omega_{m}$ & other parameters & \\
\hline$\Lambda$ CDM & 585.35 & 70.262 & 0.276 & $\Omega_{\Lambda}=0.769, \quad \Omega_{k}=-0.045$ & \\
\hline GCG & 584.54 & 70.093 & 0.277 & $\Omega_{k}=-0.019, \quad \alpha=-0.066, \quad$ & $B_{s}=0.759$ \\
\hline PCS,$d=1$ & 588.41 & 69.52 & 0.286 & $\Omega_{k}=-0.040, \quad \alpha=-0.256$, & $B=2.067$ \\
\hline PCS,$d=2$ & 591.10 & 69.49 & 0.288 & $\Omega_{k}=-0.017, \quad \alpha=-0.372$, & $B=1.599$ \\
\hline PCS,$d=3$ & 592.18 & 69.34 & 0.288 & $\Omega_{k}=-0.027, \quad \alpha=-0.431$, & $B=1.461$ \\
\hline PCS,$d=6$ & 592.56 & 69.29 & 0.289 & $\Omega_{k}=-0.029, \quad \alpha=-0.493$, & $B=1.302$ \\
\hline
\end{tabular}

\section{PCS model}

The multidimensional gravitational model of I. Pahwa, D. Choudhury and T.R. Seshadri [46] has the set of model parameters $H_{0}, \Omega_{b}, \Omega_{m}, \Omega_{k}, \alpha, B$ similar to the GCG model, but also it has the additional integer-valued parameter $d$ (the number of extra dimensions). Our analysis demonstrated that the value $d=1$ is the most preferable for describing the observational data for supernovae, BAO and $H(z)$.

So it is the case $d=1$ that we present in almost all panels of Fig. 4 (except for 2 panels with dependencies of $\min \chi_{\Sigma}^{2}$ on $H_{0}$ and $\Omega_{k}$ ). We use the similarity of model parameters for the GCG and PCS models draw in Fig. 4 the same graphs and level lines for the PCS model as in Fig. 3 in correspondent panels. Colors of correspondent lines also coincide. Naturally we use in Fig. 4 the value $B$ instead of $B_{s}$.

The minimum $\min \chi_{\Sigma}^{2}$ (over all other parameters) increases when the baryon fraction $\Omega_{b}$ grows. This dependence is more distinct than in the GCG case (Fig. 3), but it is also rather weak for small $\Omega_{b}$. So for the multidimensional model PCS we also fix $\Omega_{b}=0.047$ and really use only 5 remaining parameters $H_{0}, \Omega_{m}, \Omega_{k}, \alpha, B$. The value $\Omega_{b}=0.047$ is fixed in all panels of Fig. 4 like for Fig. 3 (except for 3 bottom-right panels).

The dependence of $\min \chi_{\Sigma}^{2}=\min _{\Omega_{m}, \Omega_{k}, \alpha, B} \chi_{\Sigma}^{2}$ on $H_{0}$ has the distinct minimum at $H_{0} \simeq 69.52$ for $d=1$ (the solid blue line here and in panels of this row). The similar behavior takes place for $d=2$ (the violet dashed line) and for $d=6$ (the purple dots). The minimal value $\min \chi_{\Sigma}^{2} \simeq 588.41$ 
for $d=1$ is larger than for the $\Lambda$ CDM and GCG models and for $d \geq 2$ the minima are still worse (see Table II).
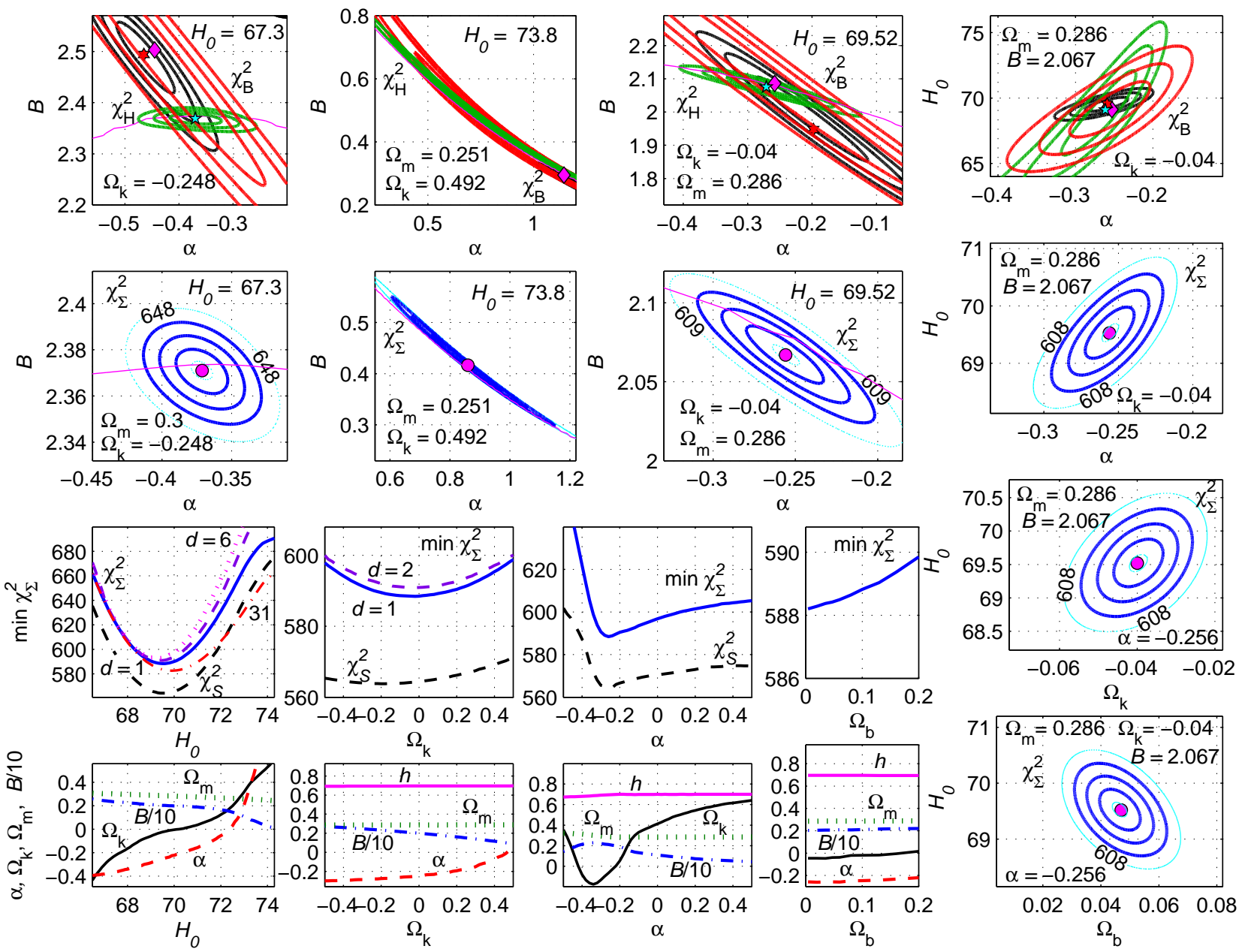

FIG. 4: The PCS model with $d=1$. Notations and panels correspond to Fig. 3, in particular, in the bottom-left panels we analyze dependence of $\min \chi_{\Sigma}^{2}$ and parameters of a minimum point on $H_{0}, \Omega_{k}, \alpha$ and $\Omega_{b}$.

These bad results for the PCS model are connected with description of the $H(z)$ recent data with high $z(z>2$ in TableVI). When we excluded 3 data points [14, 19, 20] for $H(z)$ with $z \geq 2.3$, we obtained absolutely other results presented below in Table IV.

In Fig. 4 all level lines and graphs correspond to the whole $H(z)$ data with $N_{H}=34$ points. But only one except is done for the dependence of $\min \chi_{\Sigma}^{2}$ on $H_{0}$ for $d=1$ : here $N_{H}=31$, this graph is shown as the red dash-and-dot line. The minimum value for this line $\min \chi_{\Sigma}^{2} \simeq 582.68$ is in Table IV.

Level lines of functions $\chi^{2}$ are shown in Fig. 4 in the same panels as for the GCG model in Fig. 3, in particular, for the values (37) $H_{0}=67.3,73.8$ and the optimal value $69.52 \mathrm{~km} \mathrm{c}^{-1} \mathrm{Mpc}^{-1}$. If $H_{0}$ is too large, the domain of acceptable level of $\chi_{\Sigma}^{2}$ becomes very narrow. One should note that for all level lines we change only two parameters, all remaining model parameters are fixed (they are from Table I or optimal for a given $H_{0}$ ).

In 6 top-left panels with the $\alpha, B$ plane we draw thin purple lines bounding the domain of regular solutions (below these lines). The upper domain (for larger $B$ ) consists of singular solutions, they 
have singularities in the past with infinite value of density $\rho$ corresponding to nonzero value of the scale factor $a$ [47]. These solutions are nonphysical and should be excluded. It is interesting that the optimal solutions in Fig. 4 and in Tables II and IV are near this border, but they are regular and describe the standard Big Bang $\rho \rightarrow \infty \Leftrightarrow a \rightarrow 0$ with dynamical compactification of extra dimensions.

\section{CONCLUSION}

We considered how the $\Lambda$ CDM, GCG and PCS models describe the observational data for type Ia supernovae, BAO and $H(z)$ [3], Tables V, VI, These observations distinctly restrict acceptable values for the Hubble constant $H_{0}$ and other parameters of the mentioned models. We used our calculations for dependance $\min \chi_{\Sigma}^{2}(p)$, where the absolute minimum (over other parameters) of the value (35) $\chi_{\Sigma}^{2}$ depend on a fixed parameter $p$. On the base of these calculations (presented partially in Figs. 2, 3, (4) we obtained the following $1 \sigma$ estimates for parameters of the $\Lambda$ CDM, GCG and PCS $(d=1)$ models:

TABLE III: $1 \sigma$ estimates of model parameters $\left(\Omega_{b}=0.047\right.$ in the GCG and PCS models).

\begin{tabular}{||l||c||c|c|ll||}
\hline Model & $\min \chi_{\Sigma}^{2}$ & $H_{0}$ & $\Omega_{k}$ & other parameters \\
\hline$\Lambda \mathrm{CDM}$ & 585.35 & $70.262 \pm 0.319$ & $-0.04 \pm 0.032$ & $\Omega_{m}=0.276_{-0.008}^{+0.009}, \quad \Omega_{\Lambda}=0.769 \pm 0.029$ \\
\hline GCG & 584.54 & $70.093 \pm 0.369$ & $-0.019 \pm 0.045$ & $\alpha=-0.066_{-0.074}^{+0.072}, \quad B_{s}=0.759_{-0.016}^{+0.015}$ \\
\hline PCS,$d=1$ & 588.41 & $69.523_{-0.350}^{+0.366}$ & $-0.04 \pm 0.045$ & $\Omega_{m}=0.286 \pm 0.010, \quad \alpha=-0.256_{-0.03}^{+0.032}$ \\
\hline
\end{tabular}

Our estimates for the $\Lambda$ CDM model are in agreement with the WMAP observational restrictions (38) on $\Omega_{m}, \Omega_{\Lambda}, \Omega_{k}$ [5], but they are in tension with the Planck data [8]. This fact is connected with too low value $H_{0}=67.3 \mathrm{~km} \mathrm{c}^{-1} \mathrm{Mpc}^{-1}$ (37) in the Planck survey [8].

For the GCG model min $\chi_{\Sigma}^{2}$ is slightly better and our limitations on $H_{0}$ and $\Omega_{k}$ in Table III are rather close to the $\Lambda \mathrm{CDM}$ case. However, if we require $\alpha \geq 0$ in accordance with Eq. (41) and Refs. [36, 37], the GCG model with the optimal value $\alpha=0$ will be reduced to the $\Lambda$ CDM model with its optimal parameters in Tables II, III and the same min $\chi_{\Sigma}^{2}$.

Values $\chi_{B}^{2}$ and $\chi_{\Sigma}^{2}$ for the GCG model essentially depend on the expression for $\Omega_{m}^{e f f}$ (39) or (40). But the optimal parameters in Table II for these expressions are rather close.

We mentioned above that the multidimensional model PCS is less effective in description of the considered observational data, and that the main problem of this model is connected with the $H(z)$ recent data with high $z(z>2)$. We excluded $3 H(z)$ data points [14, 19, 20] with $z=2.3$, 2.34, 2.36 and for remaining $N_{H}=31$ points of $H(z)$ and the same SN and BAO data from [3], Table V] we calculated min $\chi_{\Sigma}^{2}$ and optimal values of model parameters presented here in Table IV]

TABLE IV: Optimal values of model parameters for $\Omega_{b}=0.047$ and $N_{H}=31 H(z)$ data points with $z<2$.

\begin{tabular}{||l||c||c|c|ll||}
\hline Model & $\min \chi_{\Sigma}^{2}$ & $H_{0}$ & $\Omega_{m}$ & other parameters \\
\hline$\Lambda$ CDM & 583.71 & 70.12 & 0.281 & $\Omega_{\Lambda}=0.751, \quad \Omega_{k}=-0.032$ & \\
\hline GCG & 583.70 & 70.11 & 0.291 & $\Omega_{k}=-0.046, \quad \alpha=-0.028, \quad B_{s}=0.756$ \\
\hline PCS, $d=1$ & 582.68 & 69.89 & 0.281 & $\Omega_{k}=-0.114, \quad \alpha=-0.174, \quad B=2.078$ \\
\hline PCS, $d=2$ & 582.93 & 69.82 & 0.282 & $\Omega_{k}=-0.118, \quad \alpha=-0.290, \quad B=1.616$ \\
\hline PCS, $d=6$ & 583.23 & 69.78 & 0.282 & $\Omega_{k}=-0.126, \quad \alpha=-0.398, \quad B=1.291$ \\
\hline
\end{tabular}


We see that the model PCS [46] describes the reduced set of data with $z<2$ better than other models. The best fit is for $d=1$, the optimal value of $H_{0}$ close to $70 \mathrm{~km} \mathrm{c}^{-1} \mathrm{Mpc}^{-1}$.

This example demonstrates that predictions of any cosmological model essentially depend on data selection. Moreover, there is the important problem of model dependence (in addition to mutual dependence) of observational data, in particular, data in Tables V. VI.

Leaving the last problem beyond this paper, we can conclude that the considered observations of type Ia supernovae [3], BAO (Table V) and the Hubble parameter $H(z)$ (Table VI) confirm effectiveness of the $\Lambda$ CDM model, but they do not deny other models. The important argument in favor of the $\Lambda$ CDM model is its small number $N_{p}$ of model parameters (degrees of freedom). This number is part of information criteria of model selection statistics, in particular, the Akaike information criterion is [52] $A I C=\min \chi_{\Sigma}^{2}+2 N_{p}$. This criterion supports the leading position of the $\Lambda$ CDM model.

\section{Appendix A: Appendix}

TABLE V: Values of $d_{z}(z)=r_{s}\left(z_{d}\right) / D_{V}(z)$ (31) and $A(z)$ (32) with corresponding errors [5, 21, 22]

\begin{tabular}{||l|l|l|l|l|c||}
\hline$z$ & $d_{z}(z)$ & $\sigma_{d}$ & $A(z)$ & $\sigma_{A}$ & Refs \\
\hline 0.106 & 0.336 & 0.015 & 0.526 & 0.028 & {$[\underline{5}]$} \\
\hline 0.20 & 0.1905 & 0.0061 & 0.488 & 0.016 & {$[5]$} \\
\hline 0.35 & 0.1097 & 0.0036 & 0.484 & 0.016 & {$[\underline{5]}$} \\
\hline 0.44 & 0.0916 & 0.0071 & 0.474 & 0.034 & {$[21]$} \\
\hline 0.57 & 0.07315 & 0.0012 & 0.436 & 0.017 & {$[\underline{5,22]}$} \\
\hline 0.60 & 0.0726 & 0.0034 & 0.442 & 0.020 & {$[21]$} \\
\hline 0.73 & 0.0592 & 0.0032 & 0.424 & 0.021 & {$[21]$} \\
\hline
\end{tabular}

Measurements of $d_{z}(z)$ and $A(z)$ in Ref. 21] are not independent, they are described with the following elements of covariance matrices $C_{d}^{-1}=\left\|c_{i j}^{d}\right\|$ and $C_{A}^{-1}=\left\|c_{i j}^{A}\right\|$ in Eq. (34) [5, 21] :

$$
\begin{aligned}
& c_{44}^{d}=24532.1, \quad c_{46}^{d}=-25137.7, \quad c_{47}^{d}=12099.1 \text {, } \\
& c_{66}^{d}=134598.4, c_{67}^{d}=-64783.9, \quad c_{77}^{d}=128837.6 \text {; } \\
& c_{44}^{A}=1040.3, \quad c_{46}^{A}=-807.5, \quad c_{47}^{A}=336.8 \text {, } \\
& c_{66}^{A}=3720.3, \quad c_{67}^{A}=-1551.9, \quad c_{77}^{A}=2914.9 \text {. }
\end{aligned}
$$

These matrices are symmetric ones, their remaining elements are $c_{i i}=1 / \sigma_{i}^{2}, c_{i j}=0, i \neq j$.

\section{Acknowledgments}

G.S. would like to acknowledge the support of the Ministry of education and science of Russia (grant No. 1.476.2011).

[1] A.G. Riess et al., Observational Evidence from Supernovae for an Accelerating Universe and a Cosmological Constant, Astron. J. 116 (1998) 1009 astro-ph/9805201. 
TABLE VI: Values of the Hubble parameter $H(z)$ with errors $\sigma_{H}$ from Refs. [9 20]

\begin{tabular}{||l|l|l|l||l|l|l|l||}
\hline$z$ & $H(z)$ & $\sigma_{H}$ & Refs & $z$ & $H(z)$ & $\sigma_{H}$ & Refs \\
\hline 0.070 & 69 & 19.6 & {$[13]$} & 0.57 & 92.9 & 7.855 & {$[17]$} \\
\hline 0.090 & 69 & 12 & {$[9]$} & 0.593 & 104 & 13 & {$[11]$} \\
\hline 0.120 & 68.6 & 26.2 & {$[13]$} & 0.600 & 87.9 & 6.1 & {$[12]$} \\
\hline 0.170 & 83 & 8 & {$[9]$} & 0.680 & 92 & $8 ;$ & {$[11]$} \\
\hline 0.179 & 75 & 4 & {$[11]$} & 0.730 & 97.3 & 7.0 & {$[12]$} \\
\hline 0.199 & 75 & 5 & {$[11]$} & 0.781 & 105 & 12 & {$[11]$} \\
\hline 0.200 & 72.9 & 29.6 & {$[13]$} & 0.875 & 125 & 17 & {$[11]$} \\
\hline 0.240 & 79.69 & 2.65 & {$[16]$} & 0.880 & 90 & 40 & {$[10]$} \\
\hline 0.270 & 77 & 14 & {$[9]$} & 0.900 & 117 & 23 & {$[9]$} \\
\hline 0.280 & 88.8 & 36.6 & {$[13]$} & 1.037 & 154 & 20 & {$[11]$} \\
\hline 0.300 & 81.7 & 6.22 & {$[18]$} & 1.300 & 168 & 17 & {$[9]$} \\
\hline 0.350 & 82.7 & 8.4 & {$[15]$} & 1.430 & 177 & 18 & {$[9]$} \\
\hline 0.352 & 83 & 14 & {$[11]$} & 1.530 & 140 & 14 & {$[9]$} \\
\hline 0.400 & 95 & 17 & {$[9]$} & 1.750 & 202 & 40 & {$[9]$} \\
\hline 0.430 & 86.45 & 3.68 & {$[16]$} & 2.300 & 224 & 8 & {$[14]$} \\
\hline 0.440 & 82.6 & 7.8 & {$[12]$} & 2.340 & 222 & 7 & {$[19]$} \\
\hline 0.480 & 97 & 62 & {$[10]$} & 2.360 & 226 & 8 & {$[20]$} \\
\hline
\end{tabular}

[2] S. Perlmutter et al., Measurements of Omega and Lambda from 42 high redshift supernovae, Astrophys. J. 517 (1999) 565 astro-ph/9812133.

[3] N. Suzuki et al., The Hubble Space Telescope Cluster Supernova Survey: V. Improving the Dark Energy Constraints Above $z_{\dot{z}} 1$ and Building an Early-Type-Hosted Supernova Sample, Astrophys. J. 746 (2012) 85 arXiv:1105.3470.

[4] D.H. Weinberg et al., Observational Probes of Cosmic Acceleration, Physics Reports 530 (2013) 87 arXiv:1201.2434.

[5] G. Hinshaw et al., Nine-year Wilkinson Microwave Anisotropy Probe (WMAP) Observations: Cosmological Parameters Results, Astrophysical Journal Suppl. 208 (2013) 19 arXiv:1212.5226.

[6] D.J. Eisenstein et al., Detection of the baryon acoustic peak in the large-scale correlation function of SDSS luminous red galaxies, Astrophys. J. 633(2) (2005) 560 astro-ph/0501171.

[7] B.A. Reid et al., Cosmological constraints from the clustering of the Sloan Digital Sky Survey DR7 luminous red galaxies, Mon. Not. Roy. Astron. Soc. 404 (2010) 60 arXiv:0907.1659.

[8] P.A.R. Ade et al., Planck 2013 results. XVI. Cosmological parameters, arXiv:1303.5076.

[9] J. Simon, L. Verde and R. Jimenez, Constraints on the redshift dependence of the dark energy potential, Phys. Rev. D 71 (2005) 123001 astro-ph/0412269.

[10] D. Stern, R. Jimenez, L. Verde, M. Kamionkowski and S. A. Stanford, Cosmic chronometers: constraining the equation of state of dark energy. I: $H(z)$ measurements, J. of Cosmology and Astropart. Phys. 02 (2010) 008 arXiv:0907.3149.

[11] M. Moresco et al., Improved constraints on the expansion rate of the Universe up to $z 1.1$ from the spectroscopic evolution of cosmic chronometers, J. of Cosmology and Astropart. Phys. 8 (2012) 006 arXiv:1201.3609].

[12] C. Blake et al., The WiggleZ Dark Energy Survey: Joint measurements of the expansion and growth history at $z<1$, Mon. Not. Roy. Astron. Soc. 425(1) (2012) 405 arXiv:1204.3674.

[13] C. Zhang et al., Four New Observational H(z) Data From Luminous Red Galaxies Sloan Digital Sky Survey Data Release Seven, arXiv:1207.4541.

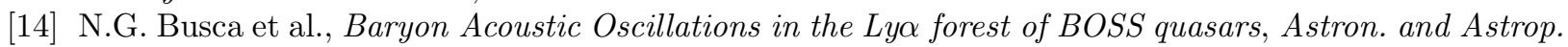
552 (2013) A96 arXiv:1211.2616. 
[15] C.H. Chuang and Y. Wang, Modeling the Anisotropic Two-Point Galaxy Correlation Function on Small Scales and Improved Measurements of $H(z), D_{A}(z)$, and $f(z) \sigma_{8}(z)$ from the Sloan Digital Sky Survey DR7 Luminous Red Galaxies, Mon. Not. Roy. Astron. Soc. 435(1) (2013) 255 arXiv:1209.0210.

[16] E. Gaztañaga, A. Cabre, L. Hui, Clustering of Luminous Red Galaxies IV: Baryon Acoustic Peak in the Line-of-Sight Direction and a Direct Measurement of H(z), Mon. Not. Roy. Astron. Soc. 399(3) (2009) 1663 arXiv:0807.3551.

[17] L. Anderson et al., The clustering of galaxies in the SDSS-III Baryon Oscillation Spectroscopic Survey: Measuring $D_{A}$ and $H$ at $z=0.57$ from the Baryon Acoustic Peak in the Data Release 9 Spectroscopic Galaxy Sample, Mon. Not. Roy. Astron. Soc. 439(1) (2014) 83 arXiv:1303.4666.

[18] A. Oka et al., Simultaneous constraints on the growth of structure and cosmic expansion from the multipole power spectra of the SDSS DR7 LRG sample, Mon. Not. Roy. Astron. Soc. 439(3) (2014) 2515 [arXiv:1310.2820.

[19] T. Delubac et al., Baryon Acoustic Oscillations in the Lyo forest of BOSS DR11 quasars, arXiv:1404.1801.

[20] A. Font-Ribera et al., Quasar-Lyman a Forest Cross-Correlation from BOSS DR11: Baryon Acoustic Oscillations, J. of Cosmology and Astroparticle Phys. 05 (2014) 027 arXiv:1311.1767.

[21] C. Blake et al., The WiggleZ dark energy survey: mapping the distance redshift relation with baryon acoustic oscillations, Mon. Not. Roy. Astron. Soc. 418(3) (2011) 1707 arXiv:1108.2635.

[22] C-H. Chuang et al., The clustering of galaxies in the SDSS-III Baryon Oscillation Spectroscopic Survey: single-probe measurements and the strong power of $f(z) \sigma_{8}(z)$ on constraining dark energy, Mon. Not. Roy. Astron. Soc. 433(4) (2013) 3559 arXiv:1303.4486.

[23] T. Clifton, P.G. Ferreira, A. Padilla and C. Skordis, Modified Gravity and Cosmology, Physics Reports 513 (2012) 1 arXiv:1106.2476.

[24] K. Bamba, S. Capozziello, S. Nojiri and S.D. Odintsov, Dark energy cosmology: the equivalent description via different theoretical models and cosmography tests, Astrophys. and Space Science 342 (2012) 155 arXiv:1205.3421.

[25] E.J. Copeland, M. Sami and S. Tsujikawa, Dynamics of dark energy, Int. J. Mod. Phys. D 15 (2006) 1753 [hep-th/0603057].

[26] M. Kunz, The phenomenological approach to modeling the dark energy, Comptes rendus - Physique 13 (2012) 539, arXiv:1204.5482.

[27] T.P. Sotiriou and V. Faraoni, $f(R)$ theories of gravity, Rev. of Modern Phys. 82 (2010) 451 arXiv:0805.1726.

[28] S. Nojiri and S. D. Odintsov, Unified cosmic history in modified gravity: from $F(R)$ theory to Lorentz non-invariant models, Phys. Rept. 505 (2011) 59 arXiv:1011.0544.

[29] R. R. Caldwell, R. Dave and P. J. Steinhardt, Cosmological imprint of an energy component with general equation of state, Phys. Rev. Lett. 80 (1998) 1582 astro-ph/9708069.

[30] J. Khoury and A. Weltman, Chameleon cosmology, Phys. Rev. D 69 (2004) 044026 astro-ph/0309411.

[31] A.Y. Kamenshchik, U. Moschella and V. Pasquier An alternative to quintessence, Phys. Lett. B 511(24) (2001) 265 arXiv:gr-qc/0103004.

[32] M.C. Bento, O. Bertolami and A.A. Sen, Generalized Chaplygin gas, accelerated expansion, and darkenergy-matter unification, Phys. Rev. D 66(4) (2002) 043507 arXiv:gr-qc/0202064].

[33] M. Makler, S.Q. de Oliveira and I. Waga, Constraints on the generalized Chaplygin gas from supernovae observations, Phys. Lett. B 555(1-2) (2003) 1 [arXiv:astro-ph/0209486.

[34] J. Lu, Y. Gui and L. Xu, Observational constraint on generalized Chaplygin gas model, Eur. Phys. J. C. 63 (2009) 349 arXiv:1004.3365.

[35] N. Liang, L. Xu and Z. Zhu, Constraints on the generalized Chaplygin gas model including gamma-ray bursts via a Markov Chain Monte Carlo approach, Astron. Astrophys. 527 (2011) A11 arXiv:1009.6059.

[36] J.P. Campos, J.C. Fabris, R. Perez, O.F. Piattella and H. Velten, Does Chaplygin gas have salvation? Eur. Phys. J. C. 73 (2013) 2357 arXiv:1212.4136.

[37] L. Xu, J. Lu and Y. Wang, Revisiting Generalized Chaplygin Gas as a Unified Dark Matter and Dark Energy Model, Eur. Phys. J. C. 72 (2012) 1883 arXiv:1204.4798.

[38] J. Lu, L. Xu, Y. Wu, and M. Liu, Combined constraints on modified Chaplygin gas model from cosmological observed data: Markov Chain Monte Carlo approach, Gen. Rel. Grav. 43 (2011) 819 arXiv:1105.1870.

[39] B.C. Paul and P. Thakur, Observational constraints on modified Chaplygin gas from cosmic growth, J. 
of Cosmology and Astropart. Phys. 11 (2013) 052 arXiv:1306.4808.

[40] N. Mohammedi, Dynamical Compactification, Standard Cosmology and the Accelerating Universe, Phys. Rev.D 65 (2002) 104018 hep-th/0202119.

[41] F. Darabi, Accelerating Universe and Dynamical Compactification of Extra Dimensions, Class. Quant. Grav. 20 (2003) 3385 gr-qc/0301075.

[42] T. Bringmann, M. Eriksson and M. Gustafsson, Cosmological Evolution of Homogeneous Universal Extra Dimensions, Phys. Rev. D 68 (2003) 063516 astro-ph/0303497.

[43] D. Panigrahi, Y.Z. Zhang and S. Chatterjee, Accelerating Universe as Window for Extra Dimensions, Int. J. Mod. Phys. A 21 (2006) 6491 gr-qc/0604079].

[44] C. A. Middleton and E. Stanley, Anisotropic evolution of 5D Friedmann-Robertson-Walker spacetime, Phys. Rev. D 84 (2011) 085013 [arXiv:1107.1828].

[45] H. Farajollahi and H. Amiri, 5D noncompact Kaluza -Klein cosmology in the presence of Null perfect fluid, Int. J. Mod. Phys. D 19 (2010) 1823 arXiv:1005.3140.

[46] I. Pahwa, D. Choudhury and T. R. Seshadri, Late-time acceleration in Higher Dimensional Cosmology, J. of Cosmology and Astroparticle Phys. 09 (2011) 015 arXiv:1104.1925.

[47] O.A. Grigorieva and G.S. Sharov, Multidimensional gravitational model with anisotropic pressure, Intern. Journal of Modern Physics D 22 (2013) 1350075 arXiv:1211.4992.

[48] A.G. Riess et al., A 3\% Solution: Determination of the Hubble Constant with the Hubble Space Telescope and Wide Field Camera 3, Astrophys. J. 730(2) (2011) 119 arXiv:1103.2976.

[49] et al. 2003, ApJ, 594, 1 J.L. Tonry et al., Cosmological Results from High-z Supernovae, Astrophys. J. 594 (2003) 1, arXiv:astro-ph/0305008

[50] . R.A. Knop et al., New Constraints on $\Omega_{m}, \Omega_{\Lambda}$, and $w$ from an Independent Set of Eleven High-Redshift Supernovae Observed with HST1, Astrophys. J. 598 (2003) 102, arXiv:astro-ph/0309368.

[51] M. Kowalski et al., Improved cosmological constraints from new, old and combined supernova datasets, Astrophys. J. 686 (2008) 749 [arXiv:0804.4142].

[52] K. Shi, Y.F. Huang and T. Lu, A comprehensive comparison of cosmological models from the latest observational data, Monthly Notices Roy. Astronom. Soc. 426 (2012) 2452 arXiv:1207.5875.

[53] O. Farooq, D. Mania and B. Ratra, Hubble parameter measurement constraints on dark energy, Astrophys. J. 764 (2013) 139 arXiv:1211.4253.

[54] O. Farooq and B. Ratra, Hubble parameter measurement constraints on the cosmological decelerationacceleration transition redshift, Astrophys. J. 766 (2013) L7, arXiv:1301.5243.

[55] O. Farooq, Ph.D. thesis, arXiv:1309.3710.

[56] J.R. Gott III, M.S. Vogeley, S. Podariu and B. Ratra, Median statistics, h0, and the accelerating universe, Astrophys. J. 549 (2001) 1. 